Supporting Information

\title{
High Internal Phase Pickering Emulsions as Templates for a Cellulosic Functional Porous Material
}

Navid Bizmark, Xiaoyu Du, and Marios A. Ioannidis ${ }^{*}$

*To whom correspondence should be addressed. Email: mioannid@uwaterloo.ca

Department of Chemical Engineering, University of Waterloo, 200 University Avenue West, Waterloo, Ontario N2L 3G1, Canada

This Supporting Information contains 4 pages and 4 figures. 


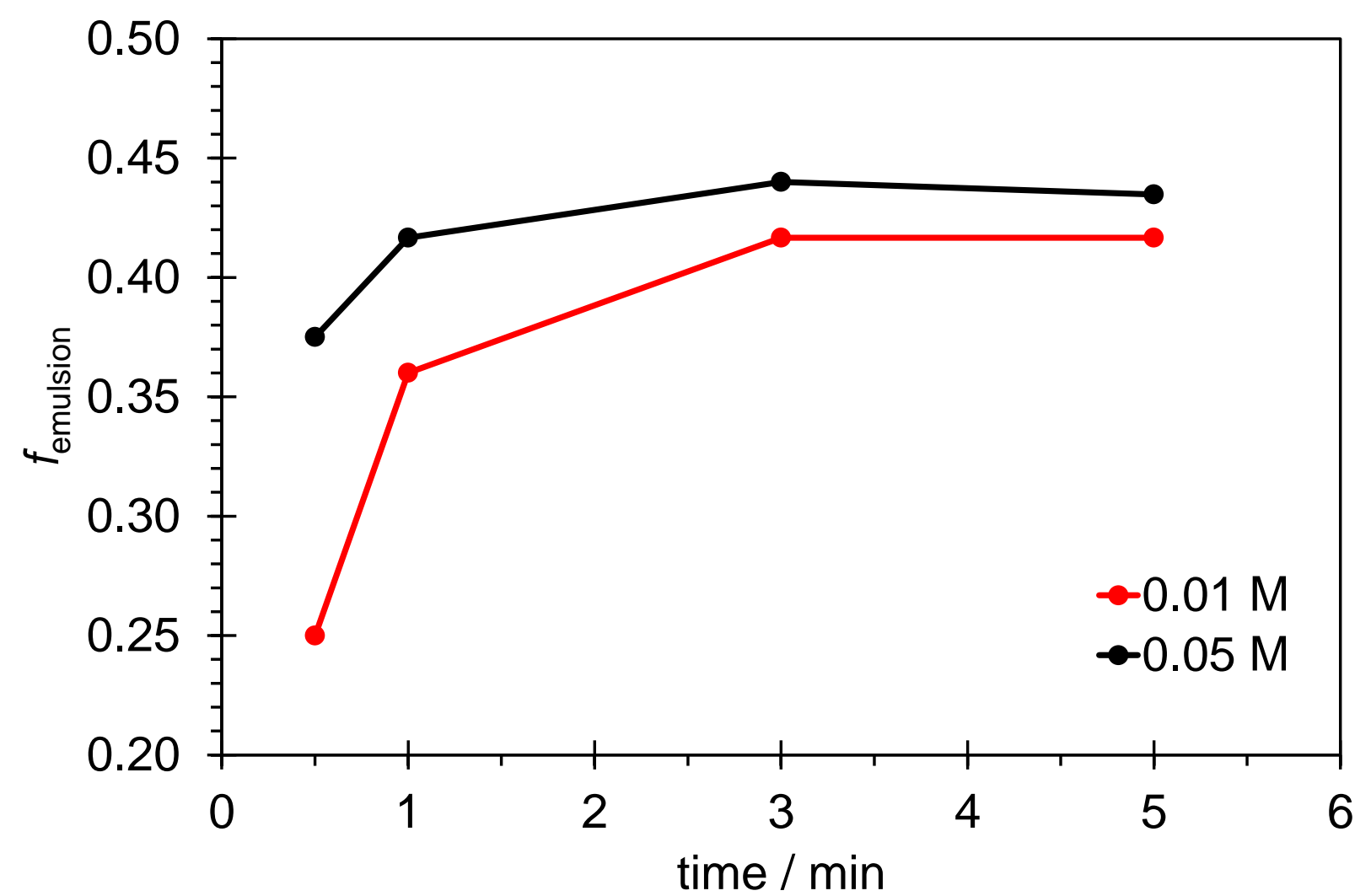

Figure S1. Volume fraction of ethyl cellulose nanoparticle-stabilized Pickering emulsions $\left(f_{\text {emulsion }} \equiv V_{\text {emulsion }} / V_{\text {total }}\right)$ generated at two different levels of ionic strength and various mixing time (30 s to $5 \mathrm{~min}$ ). Oil volume fraction, mixing speed, and nanoparticle concentration were constant at $0.33,500 \mathrm{rpm}$, and $1 \mathrm{~g} \mathrm{~L}^{-1}$, respectively. 


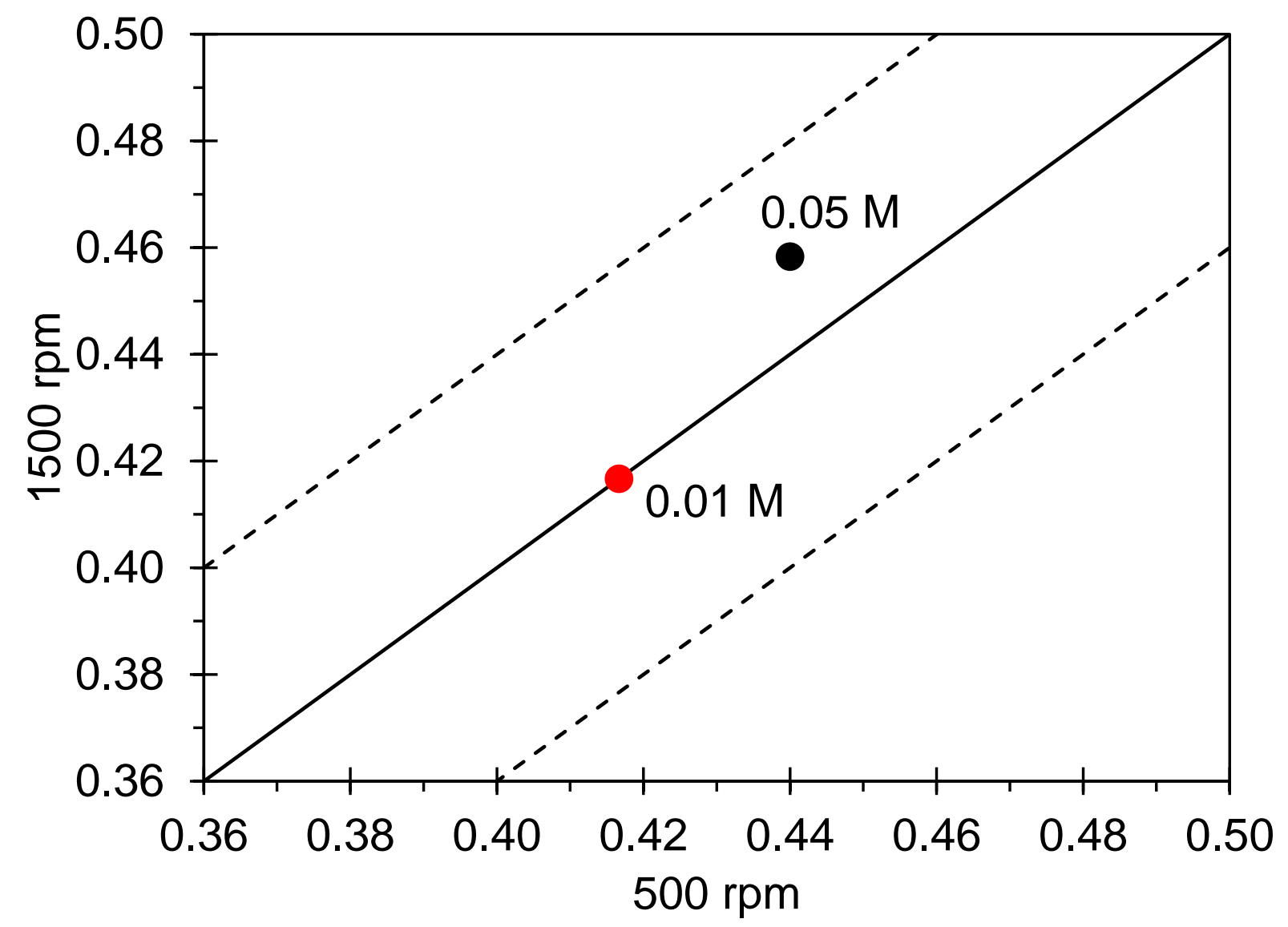

Figure S2. Volume fraction of ethyl cellulose nanoparticle-stabilized Pickering emulsions $\left(f_{\text {emulsion }} \equiv V_{\text {emulsion }} / V_{\text {total }}\right)$ generated at two different levels of ionic strength for two different mixing speeds. Mixing time, oil volume fraction, and nanoparticle concentration were constant at $3 \mathrm{~min}, 0.33$, and $1 \mathrm{~g} \mathrm{~L}^{-1}$, respectively. 


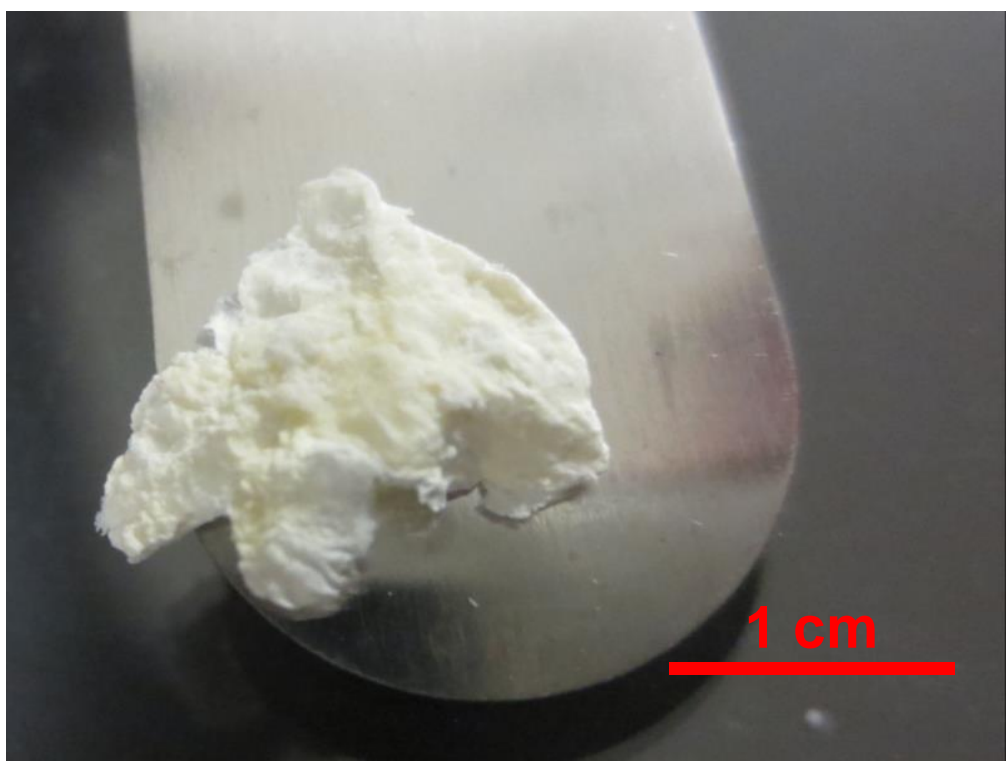

Figure S3. A porous material templated from EC nanoparticle-stabilized Pickering emulsions

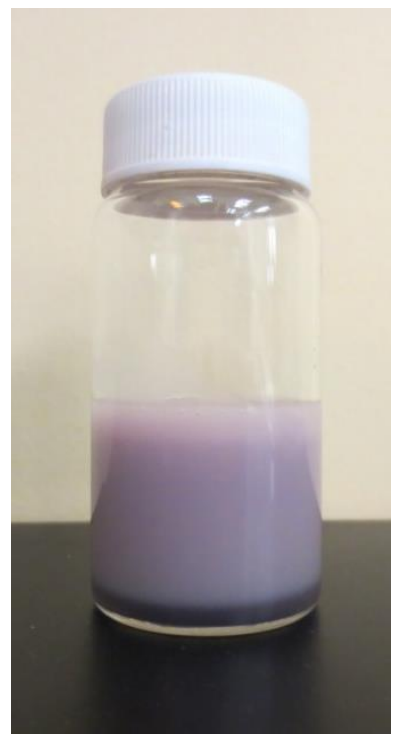

Figure S4. EC nanoparticle-stabilized high internal phase Pickering emulsion generated at $\boldsymbol{f}_{\text {oil }}=\mathbf{0 . 4 3}$, ionic strength of $0.05 \mathrm{M}$, and EC nanoparticle concentration of $1 \mathrm{~g} \mathrm{~L}^{-1}$. 


\section{Porosity estimation:}

The porosity of material $(\phi)$ shown in Figure $7(a)$ is calculated as follows:

$$
\phi=\frac{V_{\text {void }}}{V_{\text {total }}} \times 100=\frac{V_{\text {void }}}{V_{\text {solid }}+V_{\text {void }}} \times 100
$$

where $V_{\text {void }}$ is the volume of open pores and $V_{\text {solid }}$ is the volume of porous medium matrix. With reference to the video 1 in the Supporting Information, we found out that the porous material absorbed $\sim 100 \mu \mathrm{L}$ of $\mathrm{n}$-decane. We assumed that this volume indicates $V_{\text {void }}$ corresponding to open pores. $V_{\text {solid }}$ is computed from knowledge of the fact that the material was produced by emulsifying $34 \mathrm{~mL}$ of aqueous suspension of EC nanoparticles at a concentration of $10 \mathrm{~g} \mathrm{~L}^{-1}$ with $17 \mathrm{~mL}$-decane. Knowing that the density of EC is $1.14 \mathrm{~g}$ $\mathrm{mL}^{-1}$, we computed $V_{\text {solid }}=\frac{(34 \mathrm{~mL})\left(10 \frac{\mathrm{g}}{\mathrm{L}}\right)}{1.14 \frac{\mathrm{g}}{\mathrm{mL}}}=298 \mu \mathrm{L}$. Therefore, from Equation $\mathrm{S} 1, \phi=\sim 25 \%$. 